\title{
2454. Transvaginal ultrasound - noninvasive method for the prediction of response to concurrent chemoradiotherapy in cases of cervical cancer
}

\author{
J. Celiesiute ${ }^{1}$, A. Gaurilcikas ${ }^{2}$, M. Gedgaudaite ${ }^{3}$, S. Paskauskas ${ }^{4}$, K. Jariene ${ }^{5}$, D. Vaitkiene ${ }^{6}$ \\ Department of Obstetrics and Gynaecology, Medical Academy, Lithuanian University of Health Sciences, \\ Kaunas, Lithuania \\ ${ }^{1}$ Corresponding author \\ E-mail: 1joana.celiesiute@gmail.com, ${ }^{2}$ agaurilcikas@me.com, ${ }^{3}$ miglaged@gmail.com, \\ ${ }^{4}$ sauliuspaskauskas@yahoo.com, ${ }^{5}$ kristej@gmail.com, ${ }^{6}$ vaitkiene.daiva@gmail.com
}

Received 23 December 2016; received in revised form 10 April 2017; accepted 14 April 2017

DOI https://doi.org/10.21595/jve.2017.18484

Check for updates

Abstract. The objective of this paper is to study the differences in tumor size, color score and Doppler indices prior, during and after the treatment with concurrent chemoradiotherapy in cases of locally advanced cervical carcinoma and to predict the response to the treatment. The study group comprised fifty-two patients with histologically confirmed invasive carcinoma of the cervix. All patients were scheduled for concurrent chemoradiotherapy and were assessed by transvaginal ultrasound before the initiation, before 4th course of chemotherapy and 3 months after the therapy. Maximum cervical tumor length, anterior-posterior diameter and width have been measured and tumor volume was calculated. Complete clinical response (CR) was defined when no residual tumor was found. Partial clinical response (PR) was determined when the tumor volume had decreased more than $50 \%$. Intratumoral blood flow was subjectively evaluated by Color Doppler examination, the lowest resistance index (RI) and the highest peak systolic velocity (PSV) were used for the analysis. The results of this study demonstrate that transvaginal ultrasound is a valuable non-invasive diagnostic tool for the assessment of the response to concurrent chemoradiotherapy in cases of advanced cervical cancer.

Keywords: invasive cervical cancer, ultrasound, concurrent chemoradiotherapy.

\section{Introduction}

Cervical cancer is a major health problem. There were 33 thousand new cases of cervical cancer diagnosed in European Union in 2012, and 22 thousand of women died of this disease [1]. In Lithuania, the morbidity and mortality of cervical cancer is one of the highest in European Union. In the last five years, the morbidity in Lithuania remains in the interval of 25.6-29.2/ 100000 women per year. There were 431 new cases of cervical cancer in Lithuania in 2012 , and $35.5 \%$ of them were diagnosed in advanced stages. The mortality of this disease in Lithuania is 12.9/100 000 women per year, exceeding that in EU by almost three times [1,2].

The treatment strategy depends on the extension of the disease. While early stages (FIGO I-IIA) of invasive cervical cancer can be successfully treated by radical surgery, the treatment of advanced disease (from the stage II B) is complex, consisting of concurrent chemoradiotherapy (distant radiotherapy of the pelvis with concurrent chemotherapy once a week) [3].

It is quite essential to assess the effectiveness of concurrent chemoradiotherapy and to proceed with the management of cases with residual disease as soon as possible. However, the evaluation of the treatment response remains a complicated task. It has been suggested magnetic resonance imaging (MRI) to be a "gold standard" in monitoring the effectiveness of chemoradiotherapy; however, it is expensive and time-consuming method herewith in developed EU countries.

Ultrasound (US) is inexpensive, harmless method that could be carried out quickly and could be used to monitor the effectiveness of cervical cancer treatment widely as alternative method to MRI. In the grey scale, volumetric parameters of the tumour can be measured, while during Doppler examination the vascularity and blood flow (the resistance index (RI), colour score (CS), peak systolic velocity (PSV)) could be assessed. 
The aim of this study is to evaluate the differences in tumour size, colour score and Doppler indices prior, during and after the treatment with concurrent chemoradiotherapy in cases of invasive cervical carcinoma to predict the response to the treatment.

\section{Methods}

The local ethics committee approved the study (Permission No. 146/2004), and all participating patients consented to the study protocol in writing. Prospective observational study was performed. All patients diagnosed with primary advanced stage (FIGO IB2-IIIB) cervical carcinoma and treated at Lithuanian University of Health Sciences Hospital Kaunas Clinics, Gynaecology Department during the period of 2009-2011 were included in the study. The study was combined of three steps of ultrasound examination - prior the concurrent chemoradiotherapy (Step I), during the treatment - before the fourth course of chemotherapy (Step II) and three months after the treatment (Step III) (Fig. 1).

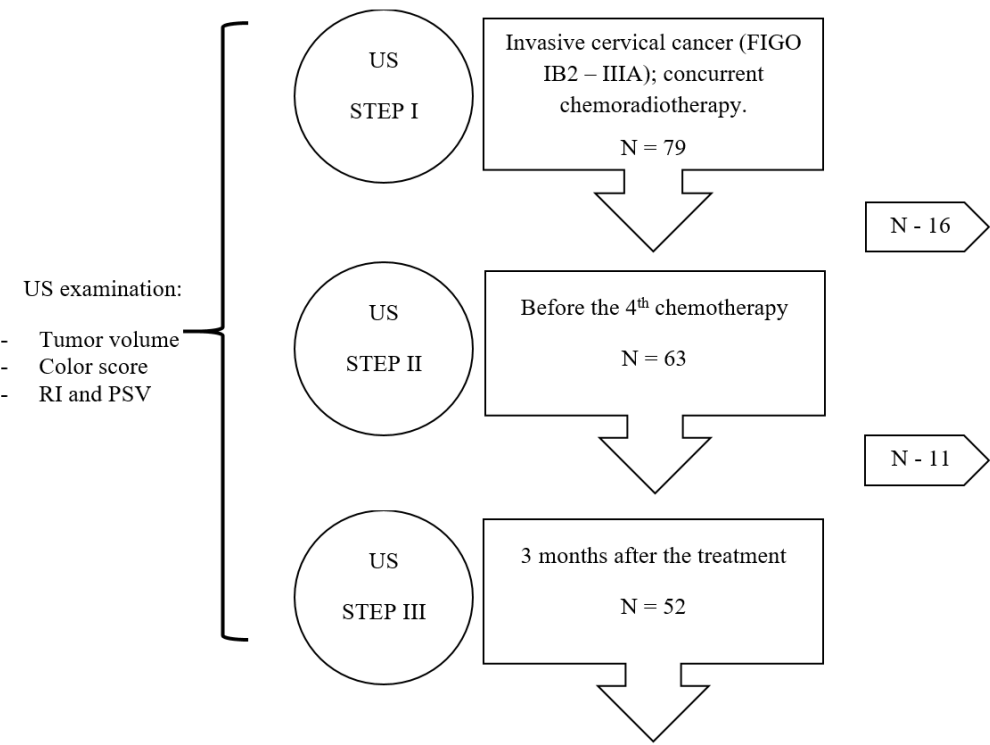

Fig. 1. Study design

The ultrasound examination was standardized. It was performed using "Toshiba Xario" ("Toshiba Medical Systems", Tokyo, Japan) ultrasound machine with transvaginal microconvex ultrasound probe Toshiba PVT-661VT (frequency range of 3.6-8.8 MHz), covered with disposable plastic cover instilled with sonogel (Parkers Inc., USA) (Fig. 2). The examination of patients consisted of two stages - grey scale examination and Doppler examination (using selected frequency of $6.0 \mathrm{MHz}$ and $7.0 \mathrm{MHz}$ ).

The patients were examined in the lithotomy position with an empty bladder. The cervical tumour was identified as an area of solid, mostly hypoechoic lesion in contrast with surrounding cervical stroma or paracervical tissue. In the sagittal plane the maximum craniocaudal length (L) and the maximum antero-posterior diameter (AP) of the tumour were measured. Then the ultrasound probe was rotated in 90 angle degree and transversal plane of the cervical tumour was obtained and the maximum width (W) was measured (Fig. 3). All morphometric characteristics of the tumour were measured minimum three times and the maximum dimensions of the tumour were analysed [7]. The volume of the tumour was calculated using formula for prolate ellipse: $\mathrm{L} \times \mathrm{AP} \times \mathrm{W} \times \pi / 6$, and expressed in $\mathrm{cm}^{3}$ [4]. The vascularity of the cervical tumour was determined by colour Doppler technique described by A. C. Testa et al. [8]. 
A

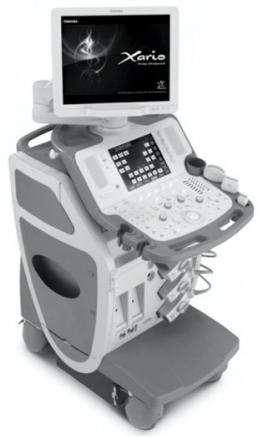

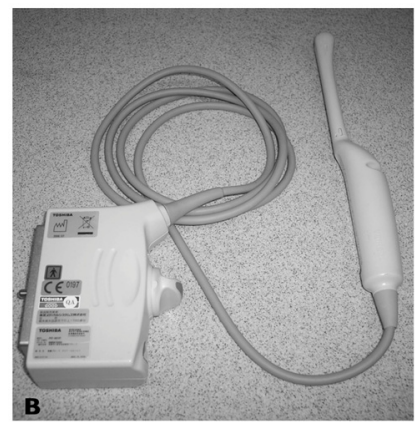

B

Fig. 2. Ultrasound equipment: a) TOSHIBA Xario XG ultrasound mashine and b) TOSHIBA PVT-661VT endovaginal Ultrasound Probe

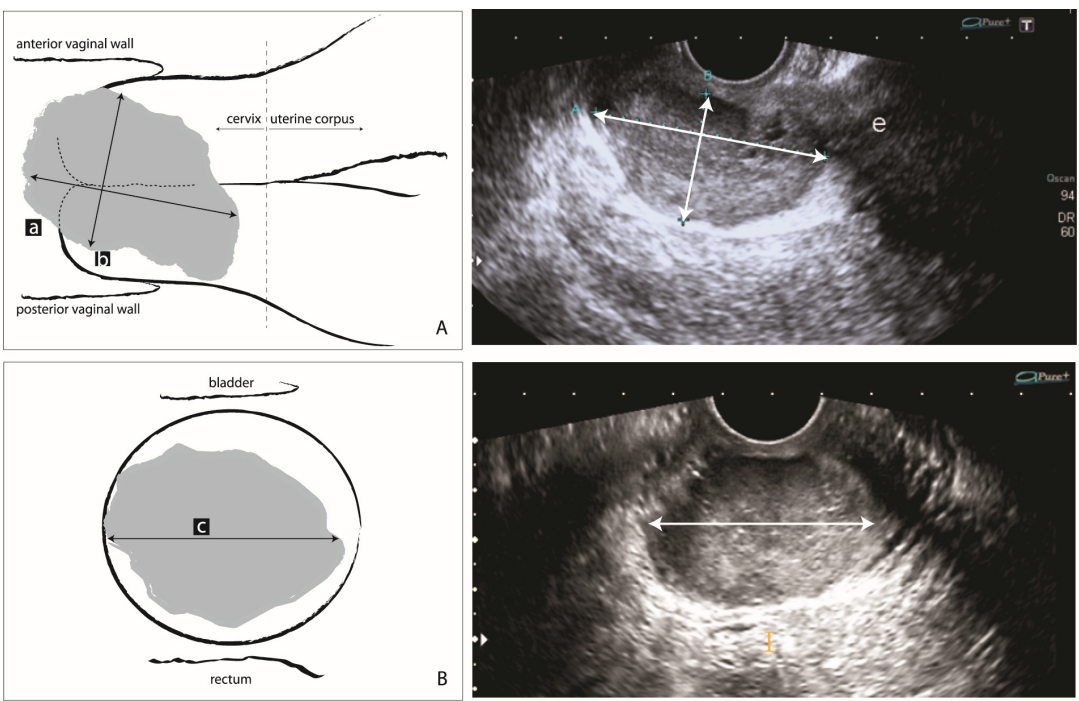

Fig. 3. Measurements of invasive cervical carcinoma: a) sagittal plane and b) transverse plane

The colour score was subjectively determined using highest sensitivity of colour Doppler signal, which allowed the detection of blood flow velocities $\geq 3 \mathrm{~cm} / \mathrm{s}$ and the colour gain was set just below the background noise level to increase the Doppler sensitivity for low-velocity flow detection. A colour score from 1 to 4 was given, 1 meaning no blood flow, 2 - minimal blood flow present in the tumour, 3 presented moderate vascularity and 4 - highly vascular tumour [8]. After the vessel was identified, the Doppler sample gate was placed over it to obtain the flow velocity waveform [5]. The resistance index (RI) was automatically calculated as the difference between peak-systolic and end-diastolic frequency shifts divided by the peak-systolic frequency shift (Fig. 4) [5, 9]. The PSV was also automatically calculated. The maximum PSV and the lowest RI found for each tumour were used in analysis.

After the Step III ultrasound examination (3 months after the chemoradiotherapy, all patients were divided into three groups (Fig. 5):

- complete response - when no residual tumour was found;

- partial response - when the tumour volume has decreased more than $50 \%$

- no response - when decrease in tumour size was less than $50 \%$.

Statistical analysis was performed using "SPSS 22.0" statistical analysis program. The $p$ value $\leq 0.05$ was considered statistically significant. Student's t-test was used for two paired samples comparison. 


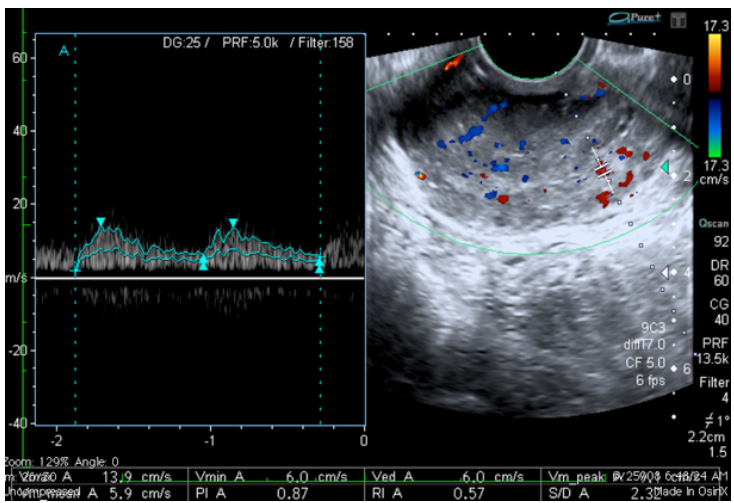

Fig. 4. Doplerometry example of tumour blood flow
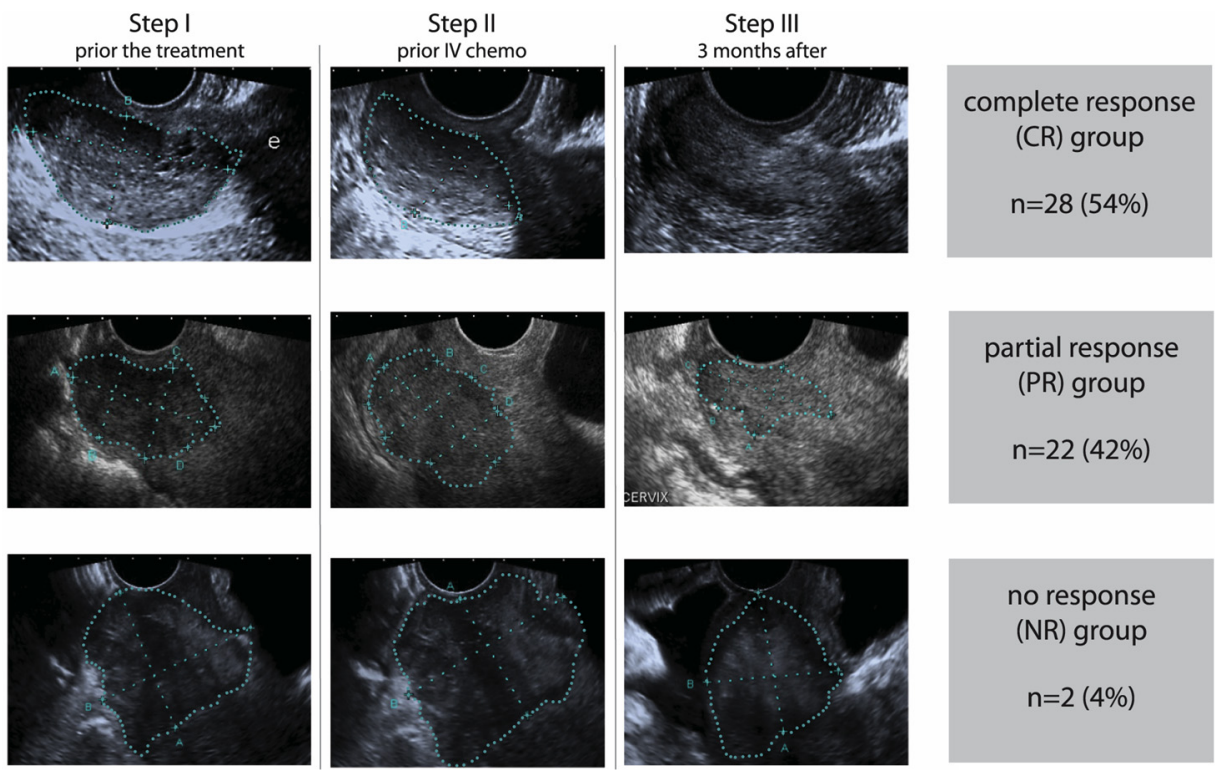

Fig. 5. Subgroups according to ultrasound findings after the treatment

\section{Results}

A total number of 79 patients were included in the study, however, 27 patients were withdrawn due to different reasons and 52 fully completed the study protocol (Fig. 2). The median age of the patients was 46 years, ranging from 28 to 76 years. 17 (32.7\%) patients were diagnosed with IB2 stage (FIGO) cervical cancer and $35(67.3 \%)$ with stage IIA or higher. Histologically, 41 (78.9\%) cases were squamous cell carcinoma, $10(19.2 \%)$ - adenocarcinoma and 1 (1.9\%) - adeno squamous carcinoma. 13 (25\%) were well differentiated tumours (Grade 1), 27 (51.9\%) moderately differentiated (Grade 2 ) and 12 (23.1\%) - poorly differentiated tumours (Grade 3 ). All main characteristics of the patients are shown in the Table 1.

CR according US examination was achieved in 28 patients (54\%), whereas $22(42 \%)$ patients demonstrated partial response. In $2(4 \%$ ) cases decrease in tumour size was less than $50 \%$ (no response group). In one of these two cases the relapse of the disease was quickly diagnosed distant metastasis was found in lungs and liver (confirmed on computer tomography). Despite the treatment, patient died in the period of six months.

The morphometric analysis showed that the mean tumour volume was not significantly different between CR and PR groups at the beginning of the treatment (Step I ultrasound exam) - 
28.1 vs. $31.3 \mathrm{~cm}^{3}, p=0.64$. Nevertheless, tumour volume in CR group was significantly lower compared with PR group (19.2 vs. 26.9, $p=0.003)$ at the middle stage of the treatment (Step II ultrasound exam) (Table 2).

The Mode of colour score before and during the treatment was 4, after the treatment -2 . A significantly lower colour score when compared to initial evaluation was found after the treatment in the group with CR $(p=0.007)$, whereas there was no difference observed in the group with PR (Fig. 6).

There was a significant difference of RI between the CR and PR groups before the treatment ( 0.53 vs. $0.68 ; p=0.045)$. Analysis of vascular indices in CR groups showed that RI after the treatment was significantly higher compared with RI before the treatment. Whereas, no significant RI changes were noted in PR group (Table 3).

Table 1. Characteristics of the patients and tumours included in this study $(n=52)$

\begin{tabular}{|l|c|}
\hline \multicolumn{1}{|c|}{ Characteristic } & Median (range) or $n(\%)$ \\
\hline Patient age (years) & $46(28-76)$ \\
\hline Stage (FIGO): & $17(32.7)$ \\
• IB2 & $35(67.3)$ \\
- IIA and higher & $41(78.9)$ \\
\hline Histology: & $10(19.2)$ \\
- Squamous cell carcinoma & $1(1.9)$ \\
- Adenocarcinoma & $13(25)$ \\
- Adeno squamous carcinoma & $27(51.9)$ \\
\hline Grade: & $12(23.1)$ \\
- G1 & \\
- G2 & \\
- G3 & \\
\hline
\end{tabular}

Table 2. Changes in tumour volume $\left(\mathrm{cm}^{3}\right)$ between the $\mathrm{CR}$ and $\mathrm{PR}$ groups during the course of treatment, mean (SD)

\begin{tabular}{|c|c|c|c|}
\hline & Before & Mid & - \\
\hline CR group & $28.1(6.8)$ & $19.2(2.2)$ & $5.7(2.7)$ \\
\hline PR group & $31.3(7.9)$ & $26.9(3.8)^{*}$ & ( \\
\hline \multicolumn{2}{|c|}{ * statistically significant difference between CR and PR groups in the mid treatment, $p=0.003$} \\
\hline
\end{tabular}

Table 3. Changes of resistance index (RI) between the CR and PR groups during the course of treatment, mean (SD)

\begin{tabular}{|c|c|c|c|}
\hline & Before & Mid & Post \\
\hline CR group & $0.53(0.19)$ & $0.61(0.21)$ & $0.79(0.11)^{*}$ \\
\hline PR group & $0.68(0.16)^{* *}$ & $0.63(0.16)$ & $0.77(0.22)$ \\
\hline *statistically significant difference of the RI before and after the treatment in the CR group, $p=0.03$ \\
**statistically significant difference of the RI between CR and PR groups before the treatment, $p=0.045$ \\
\hline
\end{tabular}

The PSV was significantly lower in CR group than in PR group before the treatment (16.6 vs. $21.3 ; p=0.04)$. After the treatment, the PSV was found significantly decreased in both groups: the CR group (8.2 vs. $16.6 ; p<0.001)$ and the PR group (10.9 vs. $21.3 ; p<0.001)$ (Table 4$)$.

Table 4. Changes of peak systolic velocity (PSV, $\mathrm{cm} / \mathrm{s}$ ) between the $\mathrm{CR}$ and $\mathrm{PR}$ groups during the course of treatment, mean (SD)

\begin{tabular}{|c|c|c|c|}
\hline & Before & Mid & Post \\
\hline CR group & $16.6(3.9)$ & $18.1(4.1)$ & $8.2(2.1)^{*}$ \\
\hline PR group & $21.3(3.2)^{* *}$ & $19.2(3.2)$ & $10.9(1.3)^{*}$ \\
\hline
\end{tabular}




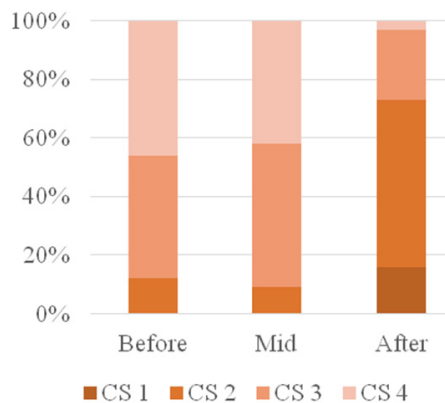

a) CR group

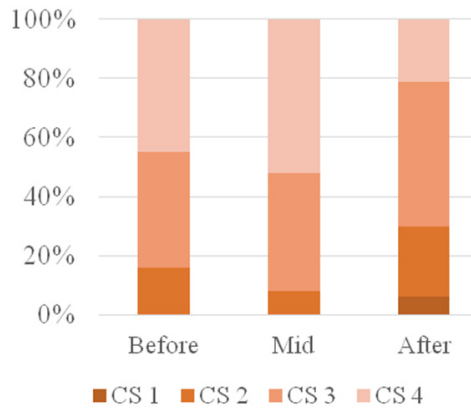

b) PER group

Fig. 6. Changes in color score (CS) during the treatment in CR and PR groups

\section{Discussion}

Until now, there is no confirmed and scientifically proven agreement on how to evaluate the effectiveness of concurrent chemoradiotherapy for cervical cancer treatment and how to diagnose the cases of inefficient treatment at the optimal time intervals. In the clinical practice the MRI and/or ultrasound are two most often used methods. Despite this fact, there is no unanimous opinion neither on capability of these methods, nor on the parameters that should be estimated to reliably monitor patients undergoing this treatment.

In our study, we compared the changes of morphometric and vascular parameters of cervical tumours during the course of concurrent chemoradiotherapy. The tumour parameters were compared between subsequent complete and partial response groups depending on the residual tumour mass seen on the ultrasound scan three months after the treatment. The latter study design was used in other studies as well [5], however it is speculative and quite precarious because cervical lesions interpreted as "residual tumour mass" on the ultrasound, were not histologically verified by performing the true-cut biopsy or hysterectomy. That is the main weakness of this study; however, it is the case in the vast majority of other studies, which have employed either ultrasound or MRI [5, 6].

The morphometric parameters of the cervical tumour were evaluated on the grey scale 2D ultrasound. In agreement with J. L. Alcazar et al., the initial tumour volume didn't differ between the subsequent complete and partial response groups [5], suggesting that the volume of primary disease has no influence on the changes of the tumour mass during proceeded concurrent chemoradiotherapy. However, in the group of complete response chemoradiotherapy results in the fast decline in tumour mass, which results in significantly lower tumour volumes and is not observed in the group of partial response. Thus, the speed of tumour mass regress should be considered as a prognostic factor. The more prominent indicator of inferior treatment effectiveness could be the identification of the residual mass after the treatment. In our study, $24(48 \%)$ cases demonstrated the "residual disease", however, we do not dare to speculate that all of them had been left with an alive tumour and were in danger to relapse. This should be confirmed by future studies.

In 1999, it was suggested by J. L. Alcazar that transvaginal Doppler ultrasound might be used to predict treatment response of concurrent chemoradiotherapy in cases of invasive cervical cancer [4]. Only few studies addressed that issue $[5,6]$ and until now there is no unanimous opinion which parameters of doplerometry are more accurate, have higher diagnostic or prognostic value. It has been observed that tumours with subsequent partial response to the therapy demonstrated higher vascularity and lower RI [4-6]. It has been suggested that the cut-off of RI 0.35 or lower could be used to predict partial response to the chemoradiotherapy [5]. Contrary, we found RI before the treatment to be significantly lower in CR compared to PR group. After the treatment, the resistance increased in both groups, however, only in CR group these changes were statistically significant. The significant difference in PSV between the groups of CR and PR before the 
treatment has been observed in our study suggesting that high PSV values could predict a poorer response to the treatment. However, this was not observed by others $[4,5]$. The discrepancies of RI and PSV studies could be explained by the complicated technique, which requires the acquisition of Doppler indices in somehow arbitrary way. We completely disagree with U. Kerimoglu et al., who proposed to use Doppler indices for the assessment of treatment response as more reliable technique than grey scale sonography or subjective assessment of colour score. It was demonstrated by our findings that remaining high colour score indices after the treatment could predict an inferior response to the chemoradiotherapy.

According to results of ultrasound examination, we defined a group of patients with local residual lesions. However, without a histological confirmation it is impossible to decide if these lesions mean a residual disease. It must be noticed that the study of similar structure should be performed where suspected residual tumour mass on ultrasound exam would be verified by histological examination. Moreover, it is an object of further studies to define the proper management of these patients. Yet, we dare to suggest that patients with suspected partial response after the treatment: residual tumour mass on grey scale ultrasound examination, highly expressed tumour vascularity (CS of 3 or 4) and low resistance indices should be taken under thorough surveillance.

Joana Celiesiute - main researcher; Adrius Gaurilcikas - main researcher; Migle Gedgaudaite - literature research and statistical part; Saulius Paskauskas - statistical work; Kristina Jariene researcher; Daiva Vaitkiene - scientific supervisor.

\section{Conclusions}

The results of our study demonstrate that transvaginal ultrasound is of value as a non-invasive diagnostic tool for the assessment of the response to concurrent chemoradiotherapy in cases of advanced cervical cancer.

\section{References}

[1] Ferlay J., Soerjomataram I., Ervik M., et al. GLOBOCAN 2012 v1.0, Cancer Incidence and Mortality Worldwide: IARC Cancer Base No. 11. International Agency for Research on Cancer, Lyon, France, 2013, http://globocan.iarc.fr.

[2] Lithuanian Cancer Registry. http://www.nvi.lt/index.php?-1414839826.

[3] National Comprehensive Cancer Network Guidelines. http://www.nccn.org/professionals/physician_gls/f_guidelines_nojava.asp.

[4] Alcazar J. L., Jurado M. Transvaginal color Doppler for predicting pathological response to preoperative chemoradiation in locally advanced cervical carcinoma: a preliminary study. Ultrasound in Medicine and Biology, Vol. 25, Issue 7, 1999, p. 1041-1045.

[5] Alcazar J. L., Castillo G., Martinez-Monge R., et al. Transvaginal color Doppler sonography for predicting response to concurrent chemoradiotherapy for locally advanced cervical carcinoma. Journal of Clinical Ultrasound, Vol. 32, Issue 6, 2004, p. 267-272.

[6] Kerimoglu U., Akata D., Hazirolan T., et al. Evaluation of radiotherapy response of cervical carcinoma with gray scale and color Doppler ultrasonography: resistive index correlation with magnetic resonance findings. Diagnostic and Interventional Radiology, Vol. 12, 2006, p. 155-160.

[7] Gaurilčikas A., Vaitkienė D., Čižauskas A., et al. Early-stage cervical cancer: agreement between ultrasound and histopathological findings with regard to tumor size and extent of local disease. Ultrasound in Obstetrics and Gynecology, Vol. 38, 2011, p. 707-715.

[8] Testa A. C., Ferrandina G., Distefano M., et al. Color Doppler velocimetry and three-dimensional color power angiography of cervical carcinoma. Ultrasound in Obstetrics and Gynecology, Vol. 24, 2004, p. 445-452.

[9] Hsieh C. Y., Wu C. C., Chen T. M., et al. Clinical significance of intratumoral blood flow in cervical carcinoma assessed by color Doppler ultrasound. Cancer, Vol. 75, Issue 10, 1995, p. 2518-2522. 


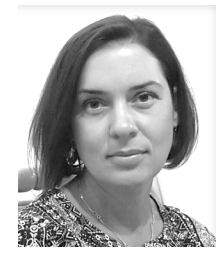

Joana Celiesiute, MD. obstetrician-gynecologist. Field of interest - gynecologic oncology, ultrasound in oncogynecology.

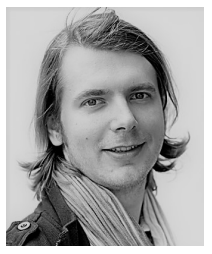

Adrius Gaurilcikas, MD, Ph.D. obstetrician-gynecologist. Field of interest - gynecologic oncology, ultrasound in oncogynecology.

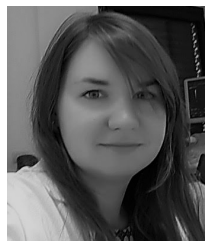

Migle Gedgaudaite, resident in obstetrics and gynecology. Field of interest - gynecologic oncology, ultrasound in oncogynecology.

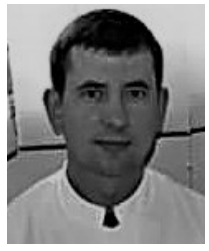

Saulius Paskauskas, MD, Ph.D., Associate Professor. Surgeon, obstetrician-gynecologist. Field of interest - gynecologic oncology, surgery, minimal invasive endoscopic surgery, minimal invasive procedures in ultrasound control.

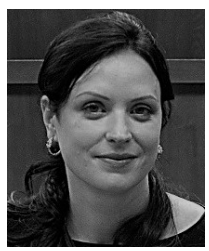

Kristina Jariene, MD, Ph.D. obstetrician-gynecologist. Field of interest - ultrasound in gynecology, gynecological endocrinology.

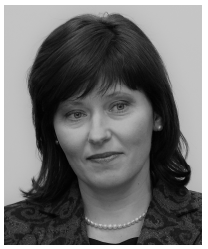

Prof. Daiva Vaitkiene, MD, Ph.D. obstetrician-gynecologist. Head of the Center of Gynecologic Oncology in Lithuanian University of Health Sciences Hospital, Kaunas Clinics. Field of interest - gynecologic oncology, ultrasound in oncogynecology, HPV infection. 